\title{
PERCEPCIÓN DEL ALUMNADO CON RESPECTO AL DESARROLLO DE LA COMPETENCIA INTERCULTURAL EN EL PROYECTO TELECOLABORATIVO ETWINNING "PREPARADOS PARA UN ERASMUS!/PRÊTS POUR UN ERASMUS!"
}

\begin{abstract}
Estibaliz de Miguel Vallés
IES Juan de Mairena

RESUMEN

En este artículo se presentan los resultados de un proyecto telecolaborativo eTwinning ${ }^{49}$ entre estudiantes de Bachillerato de francés como lengua extranjera en España y estudiantes de Bachillerato de español como lengua extranjera en Francia durante un semestre del curso académico 2015-2016. Los estudiantes se organizaron en pequeños grupos en la plataforma en línea TwinSpace intercambiando información personal y sociocultural y correcciones sobre su lengua materna (español y francés) en foros de discusión con el objetivo de mejorar su competencia intercultural.Los datos se recogieron mediante dos instrumentos que incluían los cuestionarios y las entrevistas del final del proyecto.El análisis cualitativo y cuantitativo de los datos parece indicar según la percepción de los participantes que existen evidencias del desarrollo de la competencia intercultural según el modelo de Byram (1997; 2000).
\end{abstract}

\section{PALABRAS CLAVE}

eTwinning - TwinSpace - ACAO - competencias clave - Erasmus+.

\section{ABSTRACT}

In this study, we present the findings of an on-line collaborative project eTwinning between secondary school students of French as a Foreign Language in a Spanish high school and secondary school students of Spanish as a Foreign Language in a French high school during one semester of the academic year 2015-2016. The students worked together in small groups in the online platform Twinspace, exchanging personal and socio-cultural information and corrections about each other's language (Spanish and French) in discussion forums with the aim to improve their intercultural competence. Data was gathered from two instruments that included the student's answers to a questionnaire and an interview at the end of the project. The quantitative and qualitative analysis seems to suggest regarding the participant's perception that there are evidences of development of intercultural competence as presented in Byram's model (1997; 2000).

\section{KEY WORDS}

eTwinning - TwinSpace - CSCL - key competences - Erasmus+.

\footnotetext{
49Modelo de aprendizaje colaborativo en línea. https://www.etwinning.net/es/pub/index.html
} 


\section{INTRODUCCIÓN}

El eje del proyecto eTwinning se basa en la realidad que nos encontramos al vivir en un mundo globalizado. Ya en 2013, la UNESCO publicó el informe 'Replantear la educación en un mundo en mutación'en el que describe la necesidad de replantear los principios fundamentales sobre los que se basan la educación y el aprendizaje en un mundo global.

Dentro de las consecuencias de la revolución digital, Noam Chomsky en su libro 'La Aldea Global' (2000:64) advierte sobre cómo esta revolución afectará a la desconcentración física de las aglomeraciones urbanas e industriales.

Esta revolución presenta dos realidades enfrentadas: por un lado posibilita la cooperación y la solidaridad entre personas a nivel internacional, pero por otro genera crisis relacionadas con el crecimiento del desempleo y los empleos precarios, el aumento de las desigualdades y la exclusión social.

En resumen, si la revolución digital está llevando a la transformación de la sociedad, de la economía mundial y al resto de las mutaciones sociales, esto no debería ser ignorado por la educación que, como todo lo demás, no es ajena a la globalización. García (2015:2) en su artículo sobre el estado de la cuestión III: Binomio Educación y TIC hoy precisa que "Sin duda una de las características relevantes de la sociedad en que vivimos es la incorporación de las Tecnologías de la Información y la Comunicacion (TIC) en todos los ámbitos de la vida. El entorno educativo no está aislado a esta tendencia de incorporación de las tecnologías, y vive un periodo de cambios y transformaciones que genera reflexiones y debates entre los profesionales del sector".

Si el mundo vive la tercera revolución, la digital, pero el sistema educativo sigue anclado preparando a la persona para una economía agraria o industrial, no le ofrecerá las habilidades y conocimientos que necesitará para enfrentarse de manera eficiente a los tiempos y la sociedad en la que vive y, por lo tanto, a los sistemas educativos no les queda más remedio que implementar planes de estudio innovadores y adecuados a la realidad.

Recibir una educación integrada en la revolución digital en el mundo global facilitará a las personas a responder de forma más eficiente a las demandas del mercado laboral globalizado, tan habitual por otra parte entre los graduados que suelen trabajar en países distintos al suyo y en compañías internacionales que los obligan a integrarse, organizarse y trabajar en un mundo global.

\section{LA COMPETENCIA INTERCULTURAL}

El cambio del modelo de enseñanza-aprendizaje se ajusta al nuevo contexto social, que pone el énfasis en la Competencia Comunicativa, y nos lleva hacia una dinámica intercultural.

La internacionalización de los mercados y la globalización cultural ha generado la necesidad de entablar intercambios y relaciones con personas de otros países y 
Percepción del alumnado con respecto al desarrollo de la competencia intercultural en el proyecto telecolaborativo etwinning "Preparados para un Erasmus!/Prêts pour un

Erasmus!”.

culturas. Además, el fenómeno de la migración da lugar a que las personas se vean viviendo inmersas en un entorno donde ahora existe una diversidad cultural que antes no existía y la obligación de interactuar a diario con personas procedentes de orígenes y culturas distintas.

La Unión Europea fomenta la movilidad de las personas y, en este sentido, la Comisión Europea ofrece oportunidades a los ciudadanos europeos de estudiar, hacer prácticas o volutariados en otros países de Europa a través de programas como Erasmus $+{ }^{50}$. Todo esto tiene como objetivo principal promover el conocimiento de las lenguas y culturas europeas, pero lo cierto es que el conocimiento de una lengua extranjera no garantiza que la comunicación sea eficiente entre personas procedentes de distintas culturas (Christine, 2007).

Hay que tener en cuenta que no fue hasta los años 80 que el llamado 'componente cultural' se reforzó a través de nuevas propuestas que consideraban el lenguaje como instrumento de comunicación, ampliándose el concepto de 'cultura' y gracias a la nueva relación que se estableció entre 'lengua' y 'cultura'. Gracias al hecho de empezar a considerar la competencia cultural como parte fundamental en la competencia comunicativa los individuos consiguen ser más eficaces y competentes tanto en su cultura como en la cultura de otros (Schulz et al., 2005).

Si queremos preparar a nuestros jóvenes para ser competentes en un mundo globalizado la competencia intercultural debe fomentarse en las escuelas y es necesario encontrar las maneras para evaluarla. Desde Europa oímos la voz de Van de Craen (2008: 5) que afirmó que "podemos considerar eTwinning como una apotación a la educación europea, en particular en el ámbito lingüístico y cultural. Es un claro ejemplo de cómo cooperar en un campo difícil de acceder y menos aún de cambiar. Etwinning ha demostrado que existe una educación europea y que existe para quedarse".

\section{LA COMPETENCIA INTERCULTURAL EN LOS INTERCAMBIOS COLABORATIVOS ON-LINE}

En los resultados de las investigaciones más recientes, desde O'Dowd,(2003) hasta Vinagre-(2016), se confirma que la telecoraboración en el entorno educativo promueve la Competencia Intercultural porque logra que los estudiantes se conviertan en hablantes interculturales (Müller-Hartmann, 2006; O'Dowd, 2003; 2007; Liaw, 2006; Vinagre, 2010a; Belz, 2007; Servicio Central de Apoyo eTwinning ${ }^{51}$,2014). En investigaciones como Liaw (2006), Belz (2007) o Vinagre (2008) ya se analizan estos intercambios en línea y su repercusión en el entendimiento y la empatía entre la cultura del otro y la propia y cómo se fomenta el aprendizaje intercultural.

Aun así, las investigaciones que estudian el desarrollo de la Competencia Intercultural en entornos colaborativos a distancia aún son escasas, de ahí que la evaluación de éstas siga siendo una tarea compleja para aportar más evidencias y sea

50 Véase: https://ec.europa.eu/programmes/erasmus-plus/about es

51eTwinnig es la comunidad de centros de Europa, http://etwinning.es/?lang=es 
necesario seguir realizando estudios. Por ello, este artículo lo que intenta es mostrar un adelanto de un estudio más amplio sobre este tema de investigación que a su vez, formará parte del proyecto Telecollaborative Networks for the Development of Key Competences (TELNETCOM) ${ }^{52}$ de la Universidad Autónoma de Madrid.

Este artículo, toma como punto de partida el estudio realizado por Vinagre (2014) en el que se analiza el desarrollo de la Competencia Intercultural de aprendices de lengua extranjera (español e inglés) en un intercambio telecolaborativo a través de wikis, tiene como objetivo ampliar la información en este campo de investigación a través de un modelo de aprendizaje colaborativo diferente: de páginas wiki ${ }^{53}$ al eTwinning y combinar,en este primer estudio, dos herramientas, cuestionarios y entrevistas, para que el análisis de los datos puedan dar respuesta a las siguientes preguntas:

1) A partir de la autorreflexión de los participantes, ¿se encuentran evidencias de los distintos objetivos de la competencia comunicativa intercultural según el modelo de Byram $(1997 ; 2000)$ en un proyecto e-Twinning entre estudiantes de Bachillerato?

2) En caso afirmativo, ¿qué objetivos percibe el alumno que desarrolla en mayor o menor medida en las interacciones entre los participantes?

\section{MODELO DE APRENDIZAJE DE APRENDIZAJE TELECOLABORATIVO: ETWINNING}

El modelo de aprendizaje colaborativo en línea eTwinning se lanzó en enero de 2005 como iniciativa de la Unión Europea como parte del programa eLearning para promover el modelo europeo de sociedad multilingüe y multicultural, el establecimiento de hermanamientos de centros escolares y el desarrollo de proyectos de colaboración a través de la Red. Este tipo de proyectos no tiene directrices relativas al uso de la lengua, estructura del intercambio o contenido de los temas a tratar, puesto que se basa principalmente en un trabajo de colaboración a través de las TICs entre dos o más centros escolares de países europeos que acuerdan un tema determinado del ámbito escolar.

La idea es que "dos o más centros educativos de países distintos de la Unión Europea acuerden un proyecto de trabajo conjunto, donde se apliquen las TICs para el aprendizaje colaborativo" (Moreno, 2007: 3).Los participantes de estos proyectos pueden ser el profesorado, el alumnado, las familias así como los demás miembros de la comunidad educativa.

En eTwinning tienen cabida todas las áreas, materias y familias profesionales tanto de la Educación Infantil, Primaria y Secundaria como la de las enseñanzas de régimen especial. La duración de un proyecto debe fijarse entre los socios del proyecto -no hay una fecha ni de inicio ni de fin estipulada- y los proyectos se pueden estructurar

\footnotetext{
52http://www.uamvirtual.es/TELNETCOM/

$\underline{53}$ Vinagre realizó su intercambio telecolaborativo con wikis, sitio web colaborativo que puede ser editado por varios usuarios.
} 
Percepción del alumnado con respecto al desarrollo de la competencia intercultural en el proyecto telecolaborativo etwinning "Preparados para un Erasmus!/Prêts pour un

Erasmus!”.

en dos niveles: nacional,por el Servicio Nacional de Apoyo (SNA) ${ }^{54}$ y europeo, por el Servicio Central de Apoyo (SCA ${ }^{55}$, formada por miembros de la European Schoolnet ${ }^{56} \mathrm{y}$ con sede en Bruselas.

eTwinning es la plataforma de referencia del programa Erasmus + de la KA2 ${ }^{57}$ desde 2014. Goza actualmente ${ }^{58}$ de 426.348 miembros en toda Europa involucrados en más de 54.403 proyectos activos y con 170.633 centros escolares. Sólo en España existen 2.056 proyectos activos, 12.143 centros inscritos y 33.842 docentes. Además se superan los 1000 proyectos nuevos por año.

Este éxito se debe a que los proyectos colaborativos en línea con estudiantes de diferentes países motivan enormemente a sus participantes y a que la estructura del programa facilita el proceso del intercambio porque los estudiantes tienen la posibilidad de trabajar tanto la lengua como la cultura extranjera de los otros países sin limitarse al aula y enfretándose a situaciones concretas y reales. Navracsics (2015: 5) afirma que "muchos de los jóvenes entrevistados creen que eTwinning ha sido la manera de aprender más agradable y motivadora que han tenido. Su experiencia eTwinning ha contribuido a desarrollar sus destrezas con las TIC y sus habilidades lingüísticas, así como su interculturalidad, todo ello en consonancia con los objetivos marcados cuando se presento la plataforma en el año 2005."

\section{DESCRIPCIÓN DEL PROYECTO}

Se trata de un proyecto telecolaborativo eTwinning de seis meses de duración entre estudiantes de Bachillerato de francés como lengua extranjera en España y estudiantes de Bachillerato como lengua extranjera en Francia.La muestra de participantespara este estudio es de 3 parejas, 3 hablantes nativos de francés del lycée Georges Imbert de Sarre-Union, Francia, y 3 hablantes nativos de español alumnos del IES ${ }^{59}$ Juan de Mairena de San Sebastián de los Reyes, Madrid, España, con un nivel B1 en el Marco Común Europeo de Referencia para las Lenguas: Aprendizaje, Enseñanza, Evaluación ${ }^{60}$ (2001).

Cada grupo confeccionó un blog como guía práctica para realizar un Erasmus en la ciudad de su compañero virtual. De esta manera, los alumnos se necesitaron mutuamente y colaboraron y se ayudaron para poder introducir contenidos en español

\footnotetext{
${ }^{54}$ En ingles NSS que corresponde al National Support Service

${ }^{55}$ En inglés CSS que corresponde al Cntral Support Service

${ }^{56}$ European Schoolnet es la red europea, un consorcio de 31 Ministros de educación europeos que se encargan de gestionar la acción eTwinning de la Comisión Europea y asegura su coherencia a nivel europeo.

${ }^{57}$ Las siglas KA2 corresponden a la acción clave 2 del programa Erasmus +: Cooperación para la innovación y el intercambio de buenas practices.

${ }^{58} \mathrm{https}: / /$ www.etwinning.net/es/pub/index.htm.

${ }^{59}$ Las siglas IES corresponden a Instituto de Enseñanza Secundaria.

${ }^{60}$ Corresponden a las siglas MCER o CEFR en inglés.
} 
sobre Estrasburgo y en francés sobre Madrid en sus respectivos blogs. Excepto los blogs y el diario de viaje, todas las tareas de interacción entre los alumnos presentes en los foros de discusión se realizaron en la lengua de estudio, los franceses escribieron en español y los españoles en francés.

Los estudiantes tuvieron una sesión de introducción al proyecto en la que se trataron los puntos esenciales para comenzar: descripción del proyecto, equipos de trabajo, objetivos a alcanzar, tareas a realizar, directrices a tener en cuenta desde el comienzo hasta el final de su participación y criterios de calificación.En esta sesión, los profesores se cercioraron de que los estudiantes se empezaran a sentir cómodos con el manejo de la plataforma, se resolvieron dudas y se puso en conocimiento del alumnado los criterios de evaluación y calificación: $25 \%$ de la nota total de cada evaluación (dos en total durante el semestre que duró el proyecto).

Este proyecto formó parte de la programación didáctica de ambos centros educativos, quedando los resultados registrados en la memoria final del año académico 2015-2016.

\section{TAREAS}

Una vez agrupados los participantes se les asignaron cuatro tareas y cada estudiante debía escribir en la lengua extranjera de estudio. Las tareas fueron acordadas entre los dos profesores involucrados en el proyecto y están basadas en la clasificación de Vinagre (2007: 244-245) sobre los temas de información personal (sobre sus familias, actividades diarias, estudios que realizan, donde viven, temas de interés, etc.), expresiones coloquiales, costumbres y tradiciones, música, películas de cine, arte, prejuicios y estereotipos, las fiestas y la historia.

Cada una de las tareas requería el intercambio de información y negociación de significados por parte de los estudiantes para lograr un objetivo común. Los estudiantes debieron seguir las indicaciones incluidas en la sección "Páginas" y "Foros" de la plataforma en línea, TwinSpace, y realizar las tareas con el objetivo de trabajar temas culturales de manera colaborativa.

A continuación podemos ver las secciones en las que se divide la plataforma del proyecto, TwinSpace: 
Percepción del alumnado con respecto al desarrollo de la competencia intercultural en el proyecto telecolaborativo etwinning "Preparados para un Erasmus!/Prêts pour un

Erasmus!".

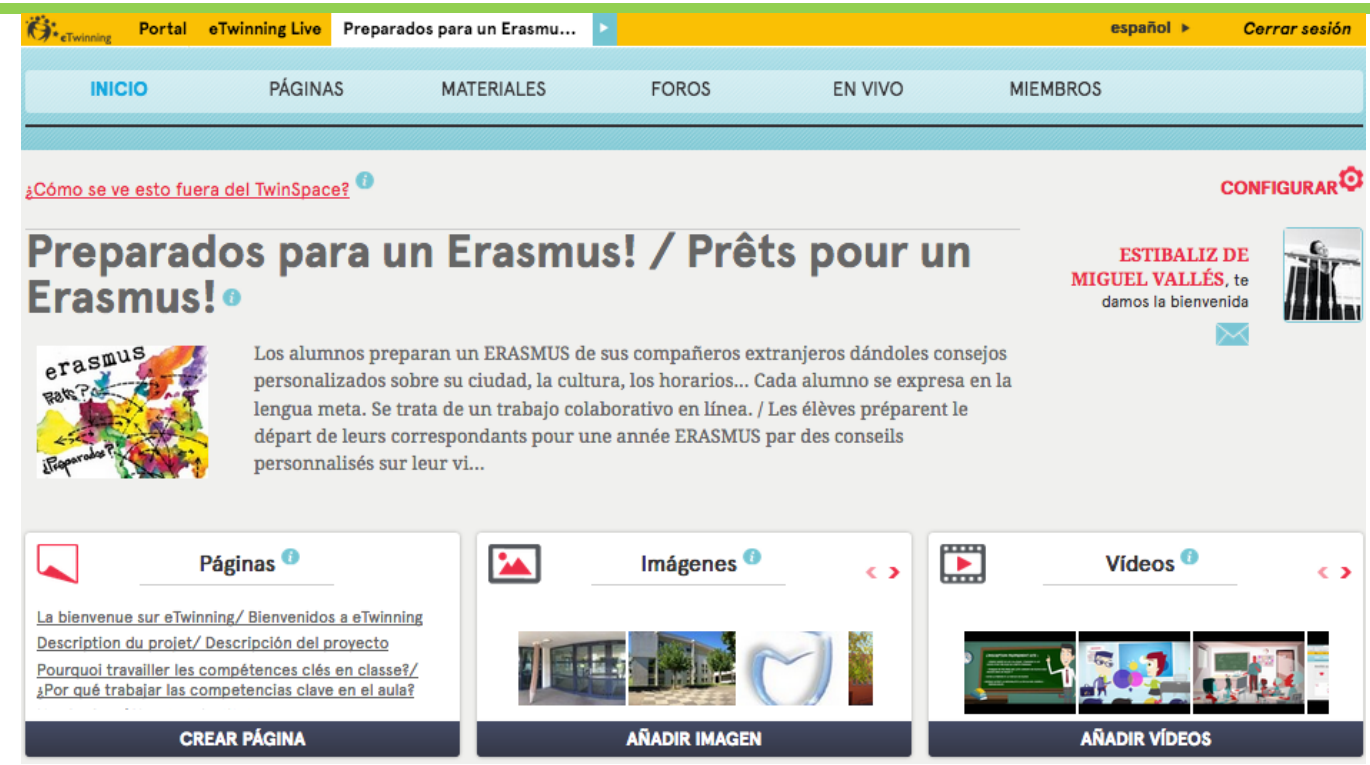

Imagen 1. Plataforma TwinSpace del proyecto eTwinning "Preparados para un erasmus/ Prêts pour un Eramus"

El éxito de la tarea dependía de la participación de todos los compañeros de la díada. Véase la descripción de las tareas en la tabla 1 :

\begin{tabular}{|c|c|}
\hline Tarea & Descripción \\
\hline $\begin{array}{l}1 \text { ¡Te presento a mi } \\
\text { compañero virtual! }\end{array}$ & $\begin{array}{l}\text { 1. Inscribíos en voki.com 2. Cread el voki o avatar de uno de vuestros } \\
\text { compañerons virtuales gracias a vuestros intercambios en el FORO de } \\
\text { Twinspace. Podéis preguntarle cómo es físicamente, lo que le gusta hacer } \\
\text { en su tiempo libre, qué tipo de música le gusta escuchar, si tiene un animal } \\
\text { de compañía, cuántos son en la familia, qué tal se lleva con ellos, etc. (8 } \\
\text { preguntas como mínimo). Es OBLIGATORIO utlizar el FORO de discusión } \\
\text { de la plataforma para interactuar y negociar lo que hace el voki de cada } \\
\text { uno, el porqué, el cómo lo váis a hacer, etc. El alumno francés se expresa } \\
\text { en todo momento en español y el alumno español en francés. 3. Colgad } \\
\text { el voki o avatar de vuestro compañero en la plataforma en línea. Cada } \\
\text { miembro del grupo debe realizar un voki o avatar utilizando la lengua } \\
\text { meta, es decir, el alumno francés tendrá que realizar el voki de su } \\
\text { compañero en español y el voki tendrá que hablar español. Del mismo } \\
\text { modo, el alumno español tendrá que realizar el voki de su compañero } \\
\text { francés y el voki tendrá que hablar francés. }\end{array}$ \\
\hline $\begin{array}{l}\text { 2. Visita al país del } \\
\text { compañero. }\end{array}$ & $\begin{array}{l}\text { Contad en el foro experiencias de cuando habéis visitado el país del } \\
\text { compañero. Podéis contar anécdotas sobre aspectos que os } \\
\text { sorprendieron, que os gustaron o no, vuestras primeras impresiones y } \\
\text { comentar sobre si estas impresiones han cambiado o no. Si no habéis } \\
\text { viajado, debéis contar lo que sabéis sobre el país del compañero y } \\
\text { vuestras imágenes preconstruidas sobre su cultura. }\end{array}$ \\
\hline
\end{tabular}




\begin{tabular}{|c|c|}
\hline $\begin{array}{l}\text { 3. ¿Qué solemos } \\
\text { hacer? }\end{array}$ & $\begin{array}{l}\text { 1. Debéis intercambiar en el foro aspectos sobre vuestro ritmo de vida, } \\
\text { la semana y el fin de semana, contando vuestros horarios, actividades... } \\
\text { Contrastad vuestro ritmo de vida personal con el de otros jóvenes que os } \\
\text { rodean. No olvidéis reaccionar a las intervenciones de vuestro } \\
\text { compañero, mostrando vuestra curiosidad y las diferencias entre los dos } \\
\text { ritmos de vida. 2. A partir de los intercambios en el Foro, escribid un texto } \\
\text { en el que anticipáis los aspectos que pueden chocar a vuestro } \\
\text { compañero cuando venga a Alsacia, así como los aspectos que le } \\
\text { parecerán normales, siendo el objetivo llevar una reflexión sobre los } \\
\text { diferentes ritmos de vida. } 3 \text {. Con los textos de todos los miembros del } \\
\text { equipo, realizad un documento Google Compartido siguiendo el modelo } \\
\text { que encontraréis en el espacio "Materiales/Documentos/Lo que solemos } \\
\text { hacer". 4. Publicad vuestro documento en ese mismo espacio. }\end{array}$ \\
\hline $\begin{array}{l}\text { 4. Cuando vengas a } \\
\text { Estrasburgo o a } \\
\text { Madrid. }\end{array}$ & 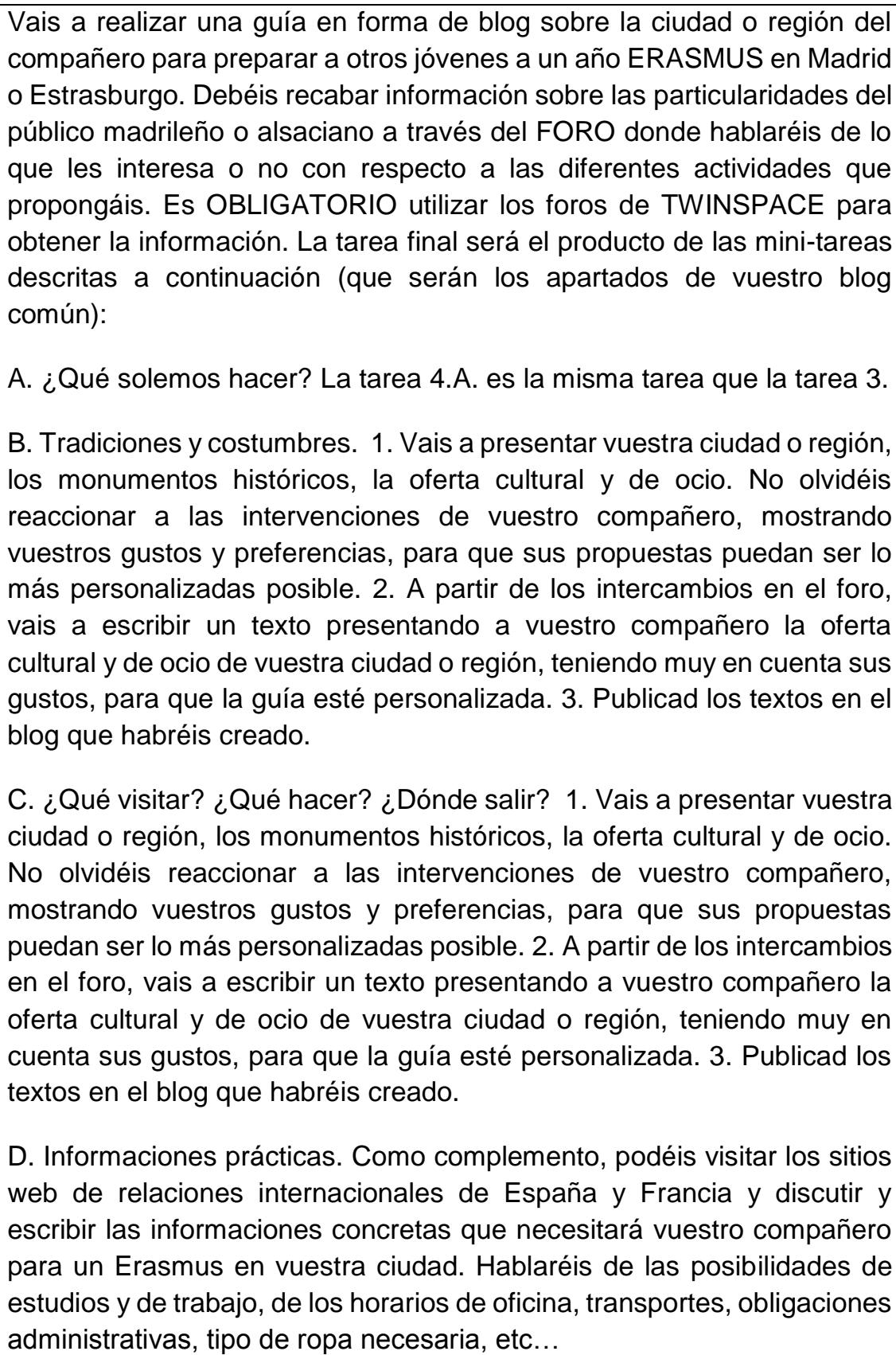 \\
\hline
\end{tabular}


Percepción del alumnado con respecto al desarrollo de la competencia intercultural en el proyecto telecolaborativo etwinning "Preparados para un Erasmus!/Prêts pour un Erasmus!".

\begin{tabular}{|l|l|}
\hline & $\begin{array}{l}\text { A partir de las informaciones recabadas en las distintas discusiones, } \\
\text { realizad un blog con los siguientes apartados: ¿Qué solemos hacer?, } \\
\text { Tradiciones y costumbres, ¿Qué visitar? ¿Qué hacer? ¿Dónde salir?, (+ } \\
\text { Informaciones prácticas). Es importante que la información lleve } \\
\text { comentarios sobre los gustos del compañero con frases como "Creo que } \\
\text { (no) te gustará..., aunque no te gusta..., ya sé que...". }\end{array}$ \\
\hline
\end{tabular}

TABLA 1. Descripción de las tareas para el intercambio de información y discusión.

\section{METODOLOGÍA}

Para contestar a las preguntas de esta investigación se analizaron las respuestas del cuestionario final de proyecto así como las respuestas a las entrevistas de final de proyecto asociadas a la percepción del alumno en su aprendizaje de la Competencia Intercultural. Para realizar la evaluación de la experiencia intercultural se adoptaron los criterios de Byram (2000:4) con algunas modificaciones basadas en Byram (1997) como parámetros para el análisis de datos. El contenido fue analizado por la autora de este artículo, responsable del proyecto eTwinning en España y, por el responsable del proyecto eTwinning en Francia ${ }^{61}$ respetando en todo momento la percepción del alumno. La construcción de los dos instrumentos, tanto en el caso del cuestionario como el de la entrevista, facilitó mucho la tarea del análisis de los datos al dividirse las preguntas según los parámetros de Byram $(1997 ; 2000)$. Dichos criterios son los siguientes (Byram, 2000:4):

a) Interés por conocer el modo de vida de otras personas y por presentar la cultura propia a otros

- Me interesan las experiencias de la vida diaria de otras personas, en especial aquellos aspectos que no suelen presentarse a los de fuera a través de los medios de comunicación.

- Estoy interesado en las experiencias diarias de una variedad de grupos sociales dentro de una sociedad y no solo de la cultura dominante.

b) Habilidad para cambiar de perspectiva

- Me doy cuenta de que soy capaz de entender otras culturas cuando miro las cosas desde otro punto de vista y cuando miro mi propia cultura desde la perspectiva de otros.

c) Conocimiento de la cultura propia y ajena para la comunicación intercultural

- Conozco algunos hechos importantes sobre la vida de la otra cultura y sobre el país, el estado y la gente.

\footnotetext{
${ }^{61}$ Miguel Ordinas Merino
} 
- Sé cómo iniciar una conversación con personas de la otra cultura y cómo mantenerla.

d) Conocimiento de (el proceso de) comunicación intercultural

- Sé cómo resolver los malentendidos que surgen cuando la gente no es consciente del punto de vista de la otra cultura.

- Sé cómo descubrir información nueva y aspectos nuevos de la otra cultura por mí mismo.

\section{RESULTADOS Y DISCUSIÓN}

Para responder a la primera pregunta de este estudio:

1) A partir de la autorreflexión de los participantes, ¿se encuentran evidencias de los distintos objetivos de la competencia comunicativa intercultural según el modelo de Byram $(1997 ; 2000)$ en un proyecto e-Twinning entre estudiantes de Bachillerato?

El análisis cualitativo de las respuestas de las entrevistas demuestran la percepción de los alumnos con respecto a si ejercitan o no todos los components de la competencia intercultural. Las aportaciones de las tablas están organizadas según la percepción del estudiante con respecto a cada componente de la competencia intercultural.

\begin{tabular}{|c|c|}
\hline Criterio/ Pregunta entrevista & Transcripciones de la pareja 1 (a1-a2) \\
\hline $\begin{array}{l}\text { Al alumno español: ¿Has mostrado } \\
\text { interés por conocer el modo de vida de } \\
\text { los franceses y por presentar la cultura } \\
\text { española a los franceses? ¿Cómo? } \\
\text { Al alumno francés: ¿Has mostrado } \\
\text { interés por conocer el modo de vida de } \\
\text { los españoles y por presentar la cultura } \\
\text { francesa a los españoles? ¿Cómo? }\end{array}$ & $\begin{array}{l}\text {-"sí hemos hablado sobre todo de cultura y gastronomía como he dicho } \\
\text { antes de cultura hemos hablado sobre Alsacia y también sobre } \\
\text { Carcassonne, que es una ciudad que está mucho más al sur de Alsacia } \\
\text { pero que también he buscado información sobre ella y también sobre } \\
\text { un castillo que hay cerca de Alsacia y sobre los cambios de horario y } \\
\text { todo este modo de vida diferente al nuestro"(trascripción a1) } \\
\text {-"oui genre euh je me montrais intéressée je lui posais des questions } \\
\text { par rapport au truc et lui il faisait de meme donc on était tous les deux } \\
\text { intéressés par les différents modes de vie de la culture de l'autre et donc } \\
\text { voilà par rapport à ce que nous on a comme traditions je lui demandais } \\
\text { si c'était pareil et au final il me disait soit que oui soit que non et si } \\
\text { vraiment c'était pas pareil il me montrait comment eux ils faisaient par } \\
\text { rapport à chez nos" (sí, me interesaba, le hacía preguntas sobre el tema } \\
\text { y él hacía lo mismo, con lo cual los dos nos interesábamos por las } \\
\text { distintas formas de vida de la cultura del otro, en cuanto a las } \\
\text { tradiciones, le preguntaba si él tenía las mismas y al final me decía que } \\
\text { sí o que no y si realmente era distinto me enseñaba cómo lo hacían ellos } \\
\text { en comparación con nosotros). (trascripción a2) }\end{array}$ \\
\hline
\end{tabular}


Percepción del alumnado con respecto al desarrollo de la competencia intercultural en el proyecto telecolaborativo etwinning "Preparados para un Erasmus!/Prêts pour un

Erasmus!”.

\begin{tabular}{|c|c|}
\hline $\begin{array}{l}\text { b) Habilidad para cambiar de } \\
\text { perspectiva } \\
\text { Al alumno español:¿Has dejado a un } \\
\text { lado tus propios valores y creencias } \\
\text { cuando intentas entender la cultura } \\
\text { francesa para cambiar de perspectiva? } \\
\text { Al alumno francés:¿Has dejado a un } \\
\text { lado tus propios valores y creencias } \\
\text { cuando intentas entender la cultura } \\
\text { española para cambiar de perspectiva? }\end{array}$ & $\begin{array}{l}\text {-"sí, yo creo que sí. Una de las cosas de las que no hemos hablado es } \\
\text { sobre el deporte, por ejemplo, y de las peleas que hay siempre entre } \\
\text { España y Francia con Nadal y Gasol. No creo que sea un tema de } \\
\text { hablar". (transcripción a1) }\end{array}$ \\
\hline $\begin{array}{l}\text { c) Conocimiento de la cultura propia y } \\
\text { ajena para la comunicación } \\
\text { intercultural } \\
\text { ¿Has mostrado a tu compañero tus } \\
\text { conocimientos sobre la cultura francesa } \\
\text { y la cultura española? }\end{array}$ & $\begin{array}{l}\text { - "sí mediante la página web primero empezamos a hablar sobre } \\
\text { diferentes cosas y al final hicimos un blog y también le enviaba fotos o } \\
\text { hablábamos por correo". (transcripción a1) } \\
\text {--------- (transcripción a2) }\end{array}$ \\
\hline $\begin{array}{l}\text { d) Conocimiento de (el proceso de) } \\
\text { comunicación intercultural } \\
\text { Al alumno español y francés: ¿Has } \\
\text { intentado solucionar los malentendidos } \\
\text { con tu compañero eTwinning? ¿Cómo? }\end{array}$ & $\begin{array}{l}\text {-"no hemos tenido malentendidos diciéndole las cosas concisas y } \\
\text { breves que sean simples y que no tuviese problemas para entenderlo". } \\
\text { (transcripción a1) } \\
\text {-"pas tujours on se comprenait pas forcément toujours sur le point de } \\
\text { vue de l'autre alors je lui demandais à lui et si vraiment je ne comprenais } \\
\text { pas j'allais regarder sur internet pour miuex comprendre quand il me } \\
\text { parlait des trucs et que je comprenais pas le mot et qu'il m'explique } \\
\text { parce qu'il explique des fois un peu voilà je comprends pas forcément } \\
\text { oui donc je regardais sur un traducteur ou un dictionnaire" (no siempre } \\
\text { no nos entendíamos siempre en cuanto al punto de vista del otro } \\
\text { entonces le preguntaba y si no lo entendía lo que me explicaba o me } \\
\text { decía buscaba en Internet para entenderle mejor porque no siempre lo } \\
\text { hacía y entonces consultaba un traductor o un diccionario. } \\
\text { (transcripción a2) }\end{array}$ \\
\hline
\end{tabular}

TABLA 2. Categorización de las trascripciones de la entrevista final pareja a1-a2

\section{\begin{tabular}{l|l} 
Tarea/Pregunta entrevista & Transcripciones de la pareja 2 (b1-b2)
\end{tabular}}

a) Interés por conocer el modo de -"yo creo que sí que he mostrado bastante interés a través de los foros vida de otras personas y por se ha visto y mediante las tareas y yo creo que por parte de mi presentar la cultura propia a otros

Pregunta en la entrevista con respecto a este criterio:

Al alumno español: $¿$ Has mostrado interés por conocer el modo de vida de los franceses y por presentar la cultura española a los franceses? ¿Cómo?

Al alumno francés: ¿Has mostrado interés por conocer el modo de vida de los españoles y por presentar la cultura francesa a los españoles? ¿Cómo? correspondiente también se la ha visto interesada por preguntarme aspectos sobre la cultura española y yo creo que también he estado preguntándole sobre la cultura francesa, sobre las dudas que tenía, informaciones que ella me ha dado"

(trascripción b1)

- "oui assez oui j'ai cherché des videos sur youtube pour regarder comment c'était surtout les fêtes qu'ils ont" (sí, bastante, he buscado vídeos en youtube para ver como eran las fiestas ahí sobretodo). (trascripción b2) 


\begin{tabular}{|c|c|}
\hline $\begin{array}{l}\text { b) Habilidad para cambiar de } \\
\text { perspectiva } \\
\text { Al alumno español: ¿Has dejado a un } \\
\text { lado tus propios valores y creencias } \\
\text { cuando intentas entender la cultura } \\
\text { francesa para cambiar de perspectiva? } \\
\text { Al alumno francés: ¿Has dejado a un } \\
\text { lado tus propios valores y creencias } \\
\text { cuando intentas entender la cultura } \\
\text { española para cambiar de perspectiva? }\end{array}$ & $\begin{array}{l}\text { "sí por ejemplo los estereotipos los hemos dejado a un lado por parte } \\
\text { de las dos aunque también los hemos dicho porque formaban parte de } \\
\text { una de las tareas pero para ver si eran verdaderos o falsos a partir de } \\
\text { ahí pues hemos sabido diferenciar por ejemplo que los españoles todo } \\
\text { el día estamos de fiesta o que somos unos vagos y esas cosas (...)" } \\
\text { (transcripción b1) } \\
\text { - "oui surtout quand elle m'a parlé de la corrida parce que je me suis dit } \\
\text { c'est célèbre en espagne du coup j'ai ensayé de comprendre pourquoi } \\
\text { je ne suis pas d'accord avec cela mais bon c'est eux qui aiment ça je } \\
\text { vais rien dire là dessus j'en ai jamais vu donc j'i pas ensayé de forcer la } \\
\text { chose" (si sobre todo cuando me ha hablado de las corrida de toros } \\
\text { pensé que era algo famoso en España y he intentado entender porque } \\
\text { estoy algo en contra pero bueno a ellos les gusta y no voy a decir nada } \\
\text { porque nunca he visto una por lo tanto no he intentado insistir en el } \\
\text { tema) (trascripción b2) }\end{array}$ \\
\hline
\end{tabular}

c) Conocimiento de la cultura propia y ajena para la comunicación intercultural

Al alumno español y francés: ¿Has mostrado a tu compañero tus conocimientos sobre la cultura francesa y la cultura española? d) Conocimiento de (el proceso de) comunicación intercultural

Al alumno español y francés: ¿Has intentado solucionar los malentendidos con tu compañero eTwinning?¿Cómo?
- "cuando tuvimos que hablar sobre los monumentos o qué visitar en Francia, pues lo típico: que conocía la Tour Eiffel, el museo del Louvre o el Arco de Triunfo, cosas así o las fiestas como el 14 de julio pero creo que lo básico no conocía mucho".

\section{(transcripción b1)}

-“ je ne connaissais pas tellement de choses sur l'Espagne pas beaucoup de traditions du coup j'ai beaucoup découvert surtout surtout sur la culture française il y avait des traditions dans le Sud de la France que je connaissais beaucoup moins mais en tout cas la culture de chez nous carrément" (no sabía tantas cosas de España de sus tradiciones por lo tanto he descubierto muchas cosas sobre la cultura francesa, había tradiciones del sur de Francia que conocía poco y he aprendido mucho de la cultura francesa en general".(transcripción b2)

-"he tenido pocos malentendidos con ella el único que recuerdo ahora es que en la última tarea que nos teníamos que poner de acuerdo en el blog y no sabíamos si yo tenía que escribir en español o en francés y ella al revés entonces yo le dije que según las instrucciones que teníamos en la página que lo mirara pero nada más"(transcripción b1)

-“ il n'y en pas eu beaucoup je pense qu'on s'est bien comprises toutes les deux donc ça n'a pas pose de problèmes quoi" (no ha habido muchos, pienso que nos hemos entendido bien las dos, no ha habido problemas) (transcripción b2)

TABLA 3. Categorización las trascripciones de la entrevista final pareja b1-b2. 


\section{Percepción del alumnado con respecto al desarrollo de la competencia intercultural en el proyecto telecolaborativo etwinning "Preparados para un Erasmus!/Prêts pour un \\ Erasmus!”.}

\begin{tabular}{|l|}
\hline Tarea/ Pregunta \\
entrevista
\end{tabular}

Al alumno francés: ¿Has mostrado interés por conocer el modo de vida de los españoles y por presentar la cultura francesa a los españoles? ¿Cómo?

b) Habilidad para cambiar de perspectiva Al alumno español:¿Has dejado a un lado tus propios valores $y$ creencias cuando intentas entender la cultura francesa para cambiar de perspectiva?

Al alumno francés:¿Has dejado a un lado tus propios valores $y$ creencias cuando intentas entender la cultura española para cambiar de perspectiva?

c) Conocimiento de la cultura propia y ajena para la comunicación intercultural ¿Has mostrado a tu compañero tus conocimientos sobre la cultura francesa y la cultura española? -"sí nos hacíamos preguntas constantes mi compañera y yo. Incluso en una tarea intercambiamos casi 140 mensajes y también nos ayudó bastante la cordialidad con la que trabajábamos". (trascripción c1)

-"euh oui j'ai essayé le plus possible de presenter notre culture objectivement et je pense qu'elle a compris en tout cas je l'espère j'ai beaucoup envoyé de photos je suis très fière de la culture culinaire que l'on a en France en Alsace et ça lui a donné très envie de goùter et de la meme manière comment dire dans la conversation ça m'a donné très envie de voyager je lui aid it que les endroits étaient très beaux et que j'avais très envie d'aller un jour en espagne à Madrid voilà" (Eh sí he intentado lo más posible presentar nuestra cultura objetivamente y pienso que ella lo ha entendido, o al menos, eso espero, le he mandado muchas fotos, estoy muy orgullosa de la cultura culinaria que tenemos en Francia, en Alsacia et le han entrado muchas ganas de probarla y de la misma manera, cómo decirlo, en nuestras conversaciones, me han entrado muchas ganas de viajar, le he dicho que los lugares que me mostraba eran muy bonitos y que tenía muchas ganas de viajar a España, à Madrid un día). (trascripción c2)

-"más o menos porque todo el mundo tiene sus propios valores, sus creencias y es difícil dejarlo de lado, pero realmente yo lo he intentado para poder entender su cultura por ejemplo la fiesta de los toros, pues ella no entendía cómo a la gente le podía gustar que matasen un toro y yo intenté explicar que es una tradición que ha existido desde hace muchos años en España y que es lo que tenemos. Que hay gente que va a estar de acuerdo con ella pero que a otra gente le gusta se lo expliqué un poco y luego ya le dije que a mí me gustaban más o menos pero que realmente a mi familia le gustaban más".(transcripción c1)

-"honnêtement non enfin je veux dire je suis restée moi-même avec mes valeurs etc mais c'est grace à ça aussi que j'ai appris à m'ouvrir en gardant mon point de vue et avec son point de vue on avait parfois des échanges avec les différents point de vue justement du coup ça ouvre notre point de vue enfin voilà" (sinceramente, no, en fin, quiero decir que sigo siendo yo misma, con mis valores, etc. Pero gracias a esto he aprendido a abrirme sin abandonar mis opiniones y ella tenía las suyas y a veces las intercambiábamos con lo cual al final nos abre más la mente) (trascripción c2)

- "Sobre la cultura francesa no he podido porque no tenía muchos. Ahí me ayudaba más ella, pero en la cultura española sí, le he puesto todo lo que sabía: monumentos, tradiciones, fiestas"(transcripción c1)

- "euh pfff honnêtement je sais pas trop si j'ai démontré mes connaissances j'ai pas mal envoyé de photos et voilà et elle aussi de la culture on a discuté". (Puff, sinceramente no sé muy bien si he demostrado mis conocimientos, le he mandado muchas fotos y hemos charlado)(transcripción c2) 


\begin{tabular}{|c|c|}
\hline $\begin{array}{l}\text { d) Conocimiento de (el } \\
\text { proceso } \\
\text { comunicación } \\
\text { intercultural } \\
\text { ¿Has intentado solucionar } \\
\text { los malentendidos con tu } \\
\text { compañero eTwinning? } \\
\text { ¿Cómo? }\end{array}$ & $\begin{array}{l}\text {-“La verdad es que no hemos tenido ningún malentendido. Ha habido un buen } \\
\text { trabajo (...) algunas veces mi compañera tardaba en responder pero porque } \\
\text { estábamos en periodo de exámenes pero finalmente acabábamos haciendo el } \\
\text { trabajo"(transcripción c1) } \\
\text {-“oui enfin dans les linguistiques dans la structure des phrases elle m'a beaucoup } \\
\text { aidee dessus moi pareil j'ai essayé de l'aider le plus possibles malgré le faiit } \\
\text { qu'elle ne faisait pas beaucoup de fautes de français je pense pas qu'il y ait eu } \\
\text { beaucoup plus de malentendus que ça on s'est toujours bien comprises". (Sí, } \\
\text { bueno, me ha ayudado mucho con la lingüística, con la estructura de las frases y } \\
\text { yo, lo mismo, he intentado ayudarla lo más que he podido aunque no cometía } \\
\text { muchos errores en francés, no pienso que haya habido muchos malentendidos, } \\
\text { creo que nos hemos comprendido bien). (transcripción c2) }\end{array}$ \\
\hline
\end{tabular}

TABLA 4. Categorización de las trascripciones de la entrevista final pareja c1-c2.

En la tabla 2, 3 y 4 se aportan las trascripciones de las 3 parejas por categorias (categorías a, b, c, d según el modelo de Byram: 2000) y se observa que la mayoría de los participantes percibe que ejercita todos los componentes de la competencia intercultural. La categoría ligeramente menos ejercitada según los participantes es la (b) "habilidad para cambiar de perspectiva". Curiosamente, el punto de vista de los participantes es que si tienen dicha habilidad; sin embargo, a1, b2 y c1 hablan de no abordar los temas conflictivos como puedan ser las corridas de toros (transcripciones de b2 y de c1) o "las peleas que hay siempre entre España y Francia con Nadal y Gasol" (trascripción de a1). Los participantes no profundizan en los temas conflictivos y se quedan en lo meramente superficial. En este caso, la percepción del alumno es diferente a la realidad, ya que no hay ni siquiera una traza de un posible cambio de perspectiva.

Para responder a la segunda pregunta de este estudio:

2) En caso afirmativo, ¿qué objetivos percibe el alumno que desarrolla en mayor o menor medida en las interacciones entre los participantes?

El análisis cuantitativo de las preguntas 13 y 14 del cuestionario final de proyecto demuestran la percepción de los alumnos con respecto a las cuatro categorías mencionadas en las directrices de Byram ${ }^{62}$ como autoreflexión sobre su experiencia en el aprendizaje de la competencia intercultural.

\footnotetext{
${ }^{62}$ Las directrices de Byram (2000) incluye una quinta además de las anteriormente citadas "ability to cope with living in a different culture" que no hemos tenido en cuenta por no ser aplicable en este proyecto.
} 
Percepción del alumnado con respecto al desarrollo de la competencia intercultural en el proyecto telecolaborativo etwinning "Preparados para un Erasmus!/Prêts pour un

Erasmus!”.

¿Qué piensas que sabes hacer?

\begin{tabular}{|l|l|l|l|}
\hline Categoría & Pregunta & Media & Desviación Típica \\
\hline \multirow{2}{*}{ A } & 1 & 4,33 & 0,47 \\
\cline { 2 - 4 } & 2 & 4,33 & 0,47 \\
\hline B & 1 & 4,00 & 0 \\
\hline \multirow{2}{*}{ C } & 1 & 3,67 & 0,47 \\
\cline { 2 - 4 } & 2 & 4,67 & 0,47 \\
\hline \multirow{2}{*}{ D } & 1 & 4,33 & 0,47 \\
\cline { 2 - 4 } & 2 & 3,67 & 0,47 \\
\hline
\end{tabular}

TABLA 5. Resultados de las categorías a, b, c y d en la pregunta 13 del cuestionario de los alumnos españoles.

La tabla muestra que los estudiantes presentan respuestas muy poco dispersas, la desviación típica es muy pequeña, luego podría considerarse que los alumnos tienen una percepción similar y alta en cuanto al desarrollo de su experiencia intercultural en todas las categorías.

¿Qué piensas que sabes hacer?

\begin{tabular}{|c|c|c|c|}
\hline Categoría & Pregunta & Media & $\begin{array}{c}\text { Desviación } \\
\text { Típica }\end{array}$ \\
\hline \multirow{2}{*}{ A } & 1 & 3,00 & 0,82 \\
\cline { 2 - 4 } & 2 & 3,33 & 0,94 \\
\hline B & 1 & 3,33 & 0,47 \\
\hline \multirow{2}{*}{ C } & 1 & 3,67 & 0,47 \\
\cline { 2 - 4 } & 2 & 3,67 & 1,25 \\
\hline \multirow{2}{*}{ D } & 1 & 3,67 & 0,94 \\
\cline { 2 - 4 } & 2 & 4,33 & 0,47 \\
\hline
\end{tabular}

TABLA 6. Resultados de las categoriás $a, b, c$ y d en la pregunta 13 del cuestionario de los alumnos franceses. 
La tabla muestra que los alumnos franceses presentan por lo general una percepción más baja que los españoles (las medias de las categorías están en su mayoría entre el 3 y 4 ).

\section{¿Qué te resulta más complicado de comprender de la cultura francesa?}

\begin{tabular}{|l|l|l|l|}
\hline Categoría & Pregunta & Media & $\begin{array}{l}\text { Desviación } \\
\text { Típica }\end{array}$ \\
\hline \multirow{2}{*}{ A } & 1 & 2,33 & 1,25 \\
\cline { 2 - 4 } & 2 & 2,67 & 1,70 \\
\hline B & 1 & 2,67 & 0,94 \\
\hline \multirow{2}{*}{ C } & 1 & 2,33 & 0,47 \\
\cline { 2 - 4 } & 2 & 2,67 & 1,70 \\
\hline \multirow{2}{*}{ D } & 1 & 3,00 & 1,41 \\
\cline { 2 - 4 } & 2 & 2,33 & 0,47 \\
\hline
\end{tabular}

TABLA 7. Resultados de las categoriás a, b, c y d en la pregunta 14 del cuestionario de los alumnos españoles.

La tabla muestra que en esta pregunta, los estudiantes españoles presentan respuestas bastante dispersas dentro de una misma pregunta (desviación típica de 1,70 en A2 y C2). En este caso, la percepción de los alumnos es más heterogénea.

¿Qué te resulta más complicado de comprender de la cultura española?

\begin{tabular}{|c|c|c|c|}
\hline Categoría & Pregunta & Media & $\begin{array}{c}\text { Desviación } \\
\text { Típica }\end{array}$ \\
\hline \multirow{2}{*}{ A } & 1 & 1,67 & 0,47 \\
\cline { 2 - 4 } & 2 & 4,00 & 0,82 \\
\hline B & 1 & 2,33 & 0,47 \\
\hline \multirow{2}{*}{ C } & 1 & 2,33 & 0,47 \\
\cline { 2 - 4 } & 2 & 2,00 & 0,82 \\
\hline \multirow{2}{*}{ D } & 1 & 2,00 & 0,82 \\
\cline { 2 - 4 } & 2 & 2,00 & 0,00 \\
\hline
\end{tabular}

TABLA 8. Resultados de las categoriás $a, b$, c y d en la pregunta 14 del cuestionario de los alumnos franceses. 
Percepción del alumnado con respecto al desarrollo de la competencia intercultural en el proyecto telecolaborativo etwinning "Preparados para un Erasmus!/Prêts pour un

Erasmus!”.

El conjunto de las respuestas a las preguntas 13 y 14 presenta menor dispersión para los franceses que para los españoles. Puede ser que se haya entendido de manera distinta la pregunta.Los alumnos españoles tuvieron una sesión presencial para realizar el cuestionario mientras que con los alumnos franceses no fue así.Se les envió el cuestionario a través del correo del TwinSpace para que lo realizan individualmente en sus casas.

Podemos concluir analizando la pregunta 13, al ser los datos más significativos que en la pregunta 14, que los alumnos españoles consideran que en las categorías $c$ y $\mathrm{d}$ hay aspectos que deberían mejorar mientras que los alumnos franceses consideran que la categía que más han desarrollado es la d.

Con respecto a la percepción del alumnado de su experiencia en este proyecto, los resultados cualitativos son muy positivos ya que consideran haber desarrollado su competencia intercultural (testimonios de las entrevistas de final de proyecto):

\section{Pareja 1}

A1:"ha sido una experiencia muy positiva ver otro sentido desde otra perspectiva tal vez hayan sido muchas cosas que hacer en muy poco tiempo junto con los exámenes pero lo hemos podido llevar adelante". (transcripción a1)/ A2:" il est bien parce que comme j'ai dit tout à l'heure on apprend plein de choses et on peut parler avec des personnes d'une autre culture donc euh c'est mieux que de parler avec des personnes qu'on côtoie tous les jours du coup on apprend à connaître de nouvelles personnes avec leur culture leurs points de vue et tout ça c'est une expérience à faire" (transcripción a2) (me gusta porque cómo he dicho antes aprendemos muchas cosas y podemos hablar con personas de otra cultura y eso es mejor que hablar con personas que tenemos a nuestro alrededor todos los días porque aprendemos a conocer a otras personas con su cultura y sus puntos de vista et es una experiencia que merece la pena).

\section{Pareja 2:}

B1: A mí me ha gustado. Me ha servido también para mejorar la lengua francesa porque aunque parezca una tontería, que no estás haciendo nada, también te ayuda bastante". (transcripción b1)/B2:"si c'était à refaire je le referais c'était super bien j'aurais bien aimé garder le contact avec ma correpondante mais on a plutôt trouvé un réseau social pour continuer à parler mais elle est sur des réseaux où moi je ne suis pas donc c'est un peu compliqué" (si tuviera que volver a hacerlo lo haría me ha encantado, me habría gustado seguir en contacto con mi intercambio pero al final decidimos buscar una red social para seguir hablando pero ella está en una red en la que yo no estoy, entonces es un poco complicado) (transcripción b2)

\section{Pareja 3}

C1:"eTwinning me parece una buena plataforma para conocer culturas, tradiciones, y mejorar el nivel de una determinada lengua, en mi caso el francés. Me parece que es una buena forma de aprender, de conocer gente nueva y me gustaría que esto se llevase más a cabo en los institutos. Me parece una mejor forma de aprender otros idiomas" (transcripción c1) 
C2: "je trouve que le projet a été bien pensé après j'aurais plutôt fait ce projet dès le début de l'annñee parce qu'à la fin tout le monde s'est un peu dissipé et s'est pas trop concentré sur le projet alors qu'au final c'était un bon projet enfin je trouvais que c'était bien organize comme je disais bien structure il y avait tout ce qu'il fallait on a présenté les camarades on a présenté la culture etc les traditions mais on aurait peut-être dû faire ça dès le début de l'année pour que tout le monde soit bien concentré là dessus et que tout le monde réussisse un bon résultat" (me parece que el proyecto se ha pensado bien pero yo lo habría llevado a cabo desde el comienzo del curso porque al final todo el mundo se ha disipado un poco y no se ha concentrado mucho en el proyecto cuando en realidad era un buen proyecto. Me pareció que estaba bien organizado, bien estructurado, tenía todo lo que tenía que tener. Hemos presentado a los compañeros, hemos presentado la cultura, etc., las tradiciones pero tendríamos que haber hecho esto desde el comienzo de curso para que todo el mundo se centrara en esto y consiguiera buenos resultados) (transcripción c2)

Otras ideas a destacar que los participantes comparten con respecto a esta experiencia son: su deseo de volver a realizar un proyecto eTwinning, su interés de aprender con un alumno nativo, estudiante y de su edad, su evidente desarrollo de la competencia intercultural, incluso conocimientos de su propia cultura (transcripción de b2) y su apertura hacia otras culturas y otra manera de ver el mundo.

\section{LIMITACIONES DEL ESTUDIO}

Las limitaciones de este estudio son, entre otras, la reducida muestra analizada que a pesar de la cantidad importante de datos obtenidos no ofrece resultados concluyentes. Para obtener resultados más significativos es necesario analizar los datos de un mayor número de estudiantes, no sólo de 3 parejas. Otra de las limitaciones es que los participantes españoles a la hora de realizar el cuestionario tuvieron una sesión presencial mientras que los participantes franceses no. Esto pudo dar lugar a que determinadas preguntas no fuesen entendidas de la misma manera por los participantes españoles y franceses. Pensamos que este hecho ha podido repercutir en los resultados obtenidos en el estudio, dando lugar a que la percepción de los alumnos españoles de su desarrollo de la competencia cultural sea mejor que la de los participantes franceses. Es posible que gracias a una sesión presencial las preguntas hubiesen sido mejor entendidas y la percepción de los franceses hubiese sido mejor (véase tabla 6). Autoras como Liaw (2006) y Vinagre (2010a) hablan de las dificultades del alumnado en cambiar de opinión y persepectiva descentrando, sabiendo ponerse en la posición del otro y logrando empatizar. En este estudio, se ha observado que el alumnado percibe en algunos casos un cambio de perspectiva, mientras que éste no es tal, ya que no se da un antes y un después evidente en sus ejemplos.

La última limitación a tener en cuenta es la referida al número de instrumentos utilizados para la recogida de datos. Deardorff (2006) y Sinicrope et al. (2007) señalaron que el uso de una combinación de herramientas es lo ideal. Por tanto, se tomarán en cuenta las sugerencias del uso de diarios de aprendizaje (O'Dowd, 2003; Vinagre 
Percepción del alumnado con respecto al desarrollo de la competencia intercultural en el proyecto telecolaborativo etwinning "Preparados para un Erasmus!/Prêts pour un

Erasmus!”.

2010b) para una triangulación de instrumentos en una futura investigación, ya que existen los diarios de viaje que los alumnos realizaron a lo largo del proyecto.

\section{CONCLUSIONES}

El objetivo primordial de este estudio fue que alumnos de Bachillerato de lengua materna español y francés reflexionasen sobre su aprendizaje de la experiencia intercultural durante un intercambio colaborativo en línea. Para evaluar este desarrollo, primeramente buscamos un modelo telecolaborativo que lo hiciese posible. El programa eTwinning nos facilitó la posibilidad de comenzar un proyecto donde se diesen las circunstancias propicias para poder evaluar los objetivos de la investigación. El proyecto elaborado por los profesores responsables tuvieron como objetivo común promover el desarrollo de la competencia intercultural de sus alumnos a través de la plataforma TwinSpace usando las lenguas de estudio.

El análisis de datos cuantitativo y cualitativo de las respuestas aportadas por los participantes a los cuestionarios y entrevistas de final de proyecto muestra que los alumnos percibieron un desarrollo de los componentes(a), (c), (d)tomando como referente las categorías del modelo de Byram (2000), es decir, consideran haber desarrollado el interés por conocer el modo de vida de los otras personas y por presentar la cultura propia a otros, el conocimiento de la cultura propia y ajena para la comunicación intercultural y el conocimiento de (el proceso) comnicación intercultural. Sin embargo, con respecto al criterio (b) perciben que no han cambiado de perspectiva y áquellos que si lo han hecho no dicen cómo, quedándose en lo superficial de los temas sin profundizar en los más conflictivos. Igualmente, con respecto a esta categoría los alumnos explican la importancia de mantener su opinión frente a la opinión del otro sin necesiad de entrar en conflicto con el compañero. Una posibilidad para evitar esto en un proyecto futuro es hacer reflexionar al alumnado en el aula sobre la cultura propia y ajena, observar las diferencias culturales, fomentar el debate y que los argumentos expuestos en el aula sean herramientas posibles de reflexión para cambiar o no de perspectiva. La percepción de cambio de perspectiva no tendría que ser visto por el alumnado como falta de personalidad o falta de criterio.Por estos motivos, las conclusiones son relativas y, se considera relevante que para una investigación futura se incluya por un lado, un número mayor de datos para que éstos sean más significativos y por otro, una combinación variada de herramientas para una mejor evaluación de la competencia intercultural.

Por último, este estudio sugiere que los intercambios colaborativos en línea a través de un proyecto eTwinning pueden ser para nuestro alumnado una herramienta útil y eficaz para el desarrollo de la competencia intercultural tanto dentro como fuera del aula. 


\section{REFERENCIAS}

Belz, J. (2003). Linguistic perspectives on the development of intercultural competence in telecollaboration. Language Learning \& Technology, 7(2), 68-117. Recuperado de http://lt.msu.edu/vol7num2/belz/

Belz, J. (2007). The development of intercultural communicative competence. On-line Intercultural Exchange: An Introduction for Foreign Language Teachers. Clevedon: Multilingual Matters. 127-166.

Byram, M. (1997). Teaching and Assessing Intercultural Communicative Competence. Clevedon, England: Multilingual Matters.

Byram, M. (2000). Assessing intercultural competence in language teaching. Sprogforum 18(6): 8-13. Recuperado de http://inet.dpb.dpu.dk/infodok/sprogforum/Espr18/byram.html

Cassells, D., Gilleran, A,. Morvan, C. \& Scimeca, S. (2015). La Generación eTwinning. Bruselas, Bélgica: Servicio Central de Apoyo eTwinning. Recuperado de https://www.etwinning.net/eun-files/generation/es.pdf

Christie, K. (2007). Learning to speak the world's languages. Phi Delta Kappan, 88(9), 645-647. https://doi.org/10.1177/003172170708800903

Chomsky, N. (2000). La aldea global (5 $5^{\mathrm{a}}$ ed.). Buenos Aires: Ed. Txalaparta S.L.

Departamento de Política Lingüística del Consejo de Europa. (2001). Marco común europeo de referencia para las lenguas: aprendizaje, enseñanza, evaluación. Recuperado de http://cvc.cervantes.es/ensenanza/biblioteca ele/marco/cvc mer.pdf

Crawley, C., Gilleran, A., Joyce, A. y Maurice, M. \& Van de Craen, P. (2008). eTwinning una aventura a través de la cultura y los idiomas. Bélgica, Bruselas. Servicio Central de Apoyo eTwinning. Recuperado de http://files.etwinning.net/shared/data/etwinning/booklet/etwinning handbook 2 008/etwinning handbook es.pdf

Gómez, M. (2015). El estado de la cuestión III: Binomio Educación y TIC hoy. EDMETIC, Revista de Educación Mediática y TIC, 4 (2): 3-6 Recuperado de https://dialnet.unirioja.es/servlet/articulo?codigo $=5192040$

Furstenberg, G., Levet, S., English, K. \& Maillet \& K. (2001). Giving virtual voice to the silent language of culture. The Cultura project. Language Learning and Technology 5 (1): 55-102. Recuperado de http://lt.msu.edu/vol5num1/furstenberg/default.html

Kearney, C. \& Gras-Velázquez, À., (2015). Una década de eTwinning: repercusiónen las prácticas, habilidades y oportunidades de desarrolloprofesional de sus docentes, contado por ellos mismos. Bruselas, Bélgica: Servicio Central de Apoyo eTwinning.

Liaw, M. (2006). E-learning and the development of intercultural competence. Language Learning and Technology, 10 (3), 49-64. 
Percepción del alumnado con respecto al desarrollo de la competencia intercultural en el proyecto telecolaborativo etwinning "Preparados para un Erasmus!/Prêts pour un

Erasmus!".

Moreno, B. (2007). La acción eTwinning: una herramienta didáctica innovadora para el aprendizaje colaborativo mediante las TICs. Revista Digital de Educación l-1 (1):1-5.

Moreno, B. (2008). La acción eTwinning: situación actual y necesidades formativas del profesorado. Revista DIM: Didáctica, Innovación y Multimedia, $12 . \quad$ Recuperado de https://dialnet.unirioja.es/servlet/articulo?codigo $=2902748$

Müller-Hartmann, A. (2006). Learning how to teach intercultural communicative competence via collaborative: A model for language teacher education. En J.A. Belz y S. Thorne (Eds.), Internet-mediated intercultural foreign language education, 63-84. Boston: Heinle \& Heinle.

O'Dowd, R. (2003). Understanding the 'other side': intercultural learning in a SpanishEnglish e-mail exchange. Language Learning and Technology, 7(2):118-144. Recuperado de http://lt.msu.edu/vol7num2/odowd/default.html

O'Dowd, R. (2013a). Telecollaborative networks in university higher education: Overcoming barriers to integration. Internet and Higher Education 18, 47-53. https://doi.org/10.1016/.i.heduc.2013.02.001

O'Dowd, R. (2013b). The competences of the telecollaborative teacher. The Language Learning Journal, 1-14.

Schultz, R. A., Lalande, J.F. I., Dyksta-Pruim, P., Zimmer-Loew, H., y James, C.J. (2005). In pursuit of cultural competence in the German language classroom: Recommandations of the AATG Task Force on the Teaching of Culture. Die Unterrichtpraxis/ Teachins German, 38 (2), 172-188.

UNESCO, (2013). Replantear la educación en un mundo en mutación. Recuperado de http://unesdoc.unesco.org/images/0022/002247/224743S.pdf

Van Ek, J.A. 1986. Objectives for Foreign Language Learning 1: Scope. Strasbourg: Council of Europe.

Vinagre, M. (2007). Integrating tandem in higher education. En O'Dowd, R. (Ed.) On-line intercultural exchange: An introduction for foreign language teachers (pp.240249) Clevedon: Multilingual Matters.

Vinagre, M. (2010). Intercultural learning in asynchronous telecollaborative exchanges: A case study. Eurocall Review, 17. Recuperado de http://www.eurocall-languages.org/review/17/index.html - vinagre

Vinagre, M. (2014). El desarrollo de la competencia intercultural en los intercambios telecolaborativos. RED (Revista de Educación a Distancia) 41, 1-21. Monográfico Interculturalidad en el nuevo paradigma educativo.

Vinagre, M. (2016). Promoting intercultural competence in culture and language studies: Outcomes of an international collaborative project. In Martín-Monje, E., Elorza, I. \& García Riaza, B. (eds.) Technological advances in specialized linguistic domains: Practical applications and mobility, 37-52. Oxon: Routledge. 
Vygotsky, L. S. (1987). Thinking and speech. In L. S. Vygotsky, collected works. New York: Plenum. 